\title{
Philosophie für Mediziner - was bringt's?
}

\section{Katharina Glatz}

Leitende Ärztin am Institut für Pathologie der Universität Basel

\section{Eine Zwischenbilanz}

Als Teilnehmerin des erstmals durchgeführten 12-tägigen Kurses «Philosophie und Medizin» der Universität Luzern ziehe ich nach den ersten zwei Kurstagen eine Zwischenbilanz.

In meiner beruflichen Tätigkeit als Pathologin habe ich Kontakt zu Medizinerinnen und Medizinern fast aller Fachrichtungen und damit die Gelegenheit, Fortbildungsveranstaltungen anderer Fachgesellschaften oder gar medizinfremder Vereinigungen zu besuchen. Im Rückblick waren es genau diese fachfremden Fortbildungen, von denen ich am meisten profitieren konnte. Aus dem neuen Blickwinkel auf scheinbar Gewohntes und Eindeutiges entstanden neue Denkmuster und daraus ergaben sich viele neue Anregungen für Weiterentwicklungen. Ein stagnierendes wissenschaftliches Projekt, durch neue Hypothesen bereichert, blühte plötzlich wieder auf.

\section{Lust auf neue Herausforderungen}

Als ich die Ausschreibung für den Kurs «Philosophie und Medizin» der Universität Luzern gesehen habe, zögerte ich zunächst. Nach dem Besuch einer Einführungsveranstaltung überwogen das Interesse und die wir alles tun sollen, was wir tun können. Die dringend notwendige gesamtgesellschaftliche Diskussion über die anzustrebenden Ziele findet aber kaum statt. Die Erarbeitung von Zielen und Sinn naturwissenschaftlicher und biomedizinischer Forschung bedürfen laut dem Existenzphilosophen Karl Jaspers philosophischer Kriterien. Diese Aufgabe kann vernünftigerweise nur in Zusammenarbeit mit Exponenten beider Fachrichtungen an die Hand genommen werden.

\section{Die Teilnehmenden}

Die zwanzigköpfige Teilnehmerschaft bringt - wenig überraschend - langjährige Berufserfahrung mit. So verschieden der Hintergrund der Teilnehmer - vom Zahnarzt über den Landarzt, den Psychiater, die Augenärztin zum Chefarzt und eben der Pathologin so ähnlich sind doch die Fragen, die uns alle bewegen: beispielsweise die Frage nach dem Status der Naturwissenschaften in der Medizin. Obwohl sich die Ansichten der Teilnehmer in puncto Wissenschaftsgläubigkeit deutlich unterscheiden, sind sie durch die Erkenntnis, dass die Menschheit trotz eindrücklicher wissenschaftlicher Fortschritte auf grundlegende Fra-

\section{Der Kurs eignet sich für jeden, der die Möglichkeit einer kritischen Reflexion seines medizinischen Alltags vermisst.}

Neugier die Hemmungen, sich mit einem geisteswissenschaftlichen Fach auseinanderzusetzen, das einem während der Gymnasialzeit lediglich dazu gedient hatte, die Hausaufgaben der anderen Fächer speditiv zu erledigen.

$\mathrm{Zu}$ dieser Lust auf eine neue Herausforderung nach über 10 Jahren praktischer und wissenschaftlicher Tätigkeiten im Bereich der Pathologie kam ein wachsendes Unbehagen über die rasante Entwicklung im Bereich der biomedizinischen Forschung, die zunehmend ausserhalb der Kontrolle des öffentlichen Diskurses stattfindet, weil nicht nur die Öffentlichkeit, sondern auch wir Mediziner in einer Flut neuer und immer komplexerer Erkenntnisse unterzugehen drohen. Parallel dazu werden die Forderungen und die Anspruchshaltung der Patientinnen und Patienten immer grösser.

\section{Medizinischer Fortschritt: wie weiter?}

Der medizinische Fortschritt ist mittlerweile an einem Punkt angelangt, an dem die Frage berechtigt ist, ob gen wie die nach der Existenz des freien Willens nach wie vor keine überzeugenden Antworten weiss und möglicherweise nie wissen wird, in Bescheidenheit zusammengerückt.

\section{Jenseits von Esoterik und Wissenschafts- gläubigkeit}

Anhand dreier Texte von Philosophen, die drei systematische Standpunkte repräsentieren, setzen wir uns am zweiten Tag mit Fragen medizinischer Anthropologie auseinander. Dank verständlicher Interpretation der Texte durch den Dozenten driften die anschliessenden Diskussionen weder ins Esoterische noch ins Szientistische ab.

Selten habe ich einen Kurs erlebt, in dem die Teilnehmerinnen und Teilnehmer über 8 Stunden pausenlos konzentriert bis zum Schluss bei der Sache waren und eifrig ihre Notizblöcke vollschrieben, als gäbe es eine Prüfung zu bestehen. Am Ende des Kurstages möchte man am liebsten weiterdiskutieren und 


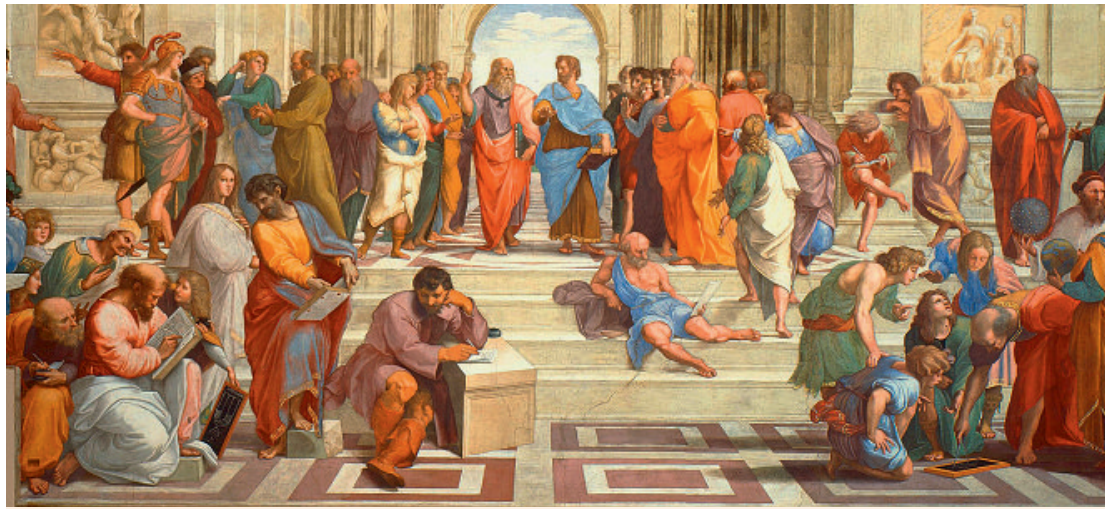

Ausschnitt aus Raffaels «Schule von Athen» mit den Gründervätern der abendländischen Philosophie. Ihre Gedanken und die ihrer Nachfolger können auch bei medizinischen Fragestellungen Orientierung geben. wähnt sich unter dem Einfluss einer bewusstseinserweiternden Droge. Eine willkommene Nebenwirkung, die dieser Kurs durchaus haben kann.

\section{Wer soll diesen Kurs besuchen?}

Der Kurs eignet sich für diejenigen, die ihr Tun gerne hinterfragen und die Möglichkeit einer kritischen Reflexion ihres medizinischen Alltags vermissen. Man sollte keine Angst haben vor dem Moment, in
Ich meine, jeder Mediziner, jede Medizinerin sollte sich einen solchen Blick über den Tellerrand gönnen. Ich selbst befinde mich fünf Jahre nach der Habilitation in der Mitte meiner beruflichen Karriere, wo es angebracht ist, eine Zwischenbilanz des Erreichten zu ziehen und sich zu überlegen, wohin es noch gehen soll und kann. Die meisten Kursteilnehmer befinden sich im letzten Drittel ihrer beruflichen Laufbahn, wodurch unweigerlich existentielle Fragen vermehrt in den Vordergrund rücken und der Erfahrungsschatz aus Beruf und Leben zur vermehrten Reflexion der eigenen Arbeit prädisponieren.

\section{Wie viel Zeit muss ich investieren?}

Insgesamt dauert der Kurs 12 Seminartage, verteilt über ein Jahr. Zwei bis drei Wochen vor dem nächsten Kurstag wird die Vorbereitungslektüre verteilt. Rund 70 Seiten sind es für den nächsten Kurstag; nicht immer ganz einfach zu lesende Texte von teils sehr bekannten, teils weniger bekannten, aber deshalb nicht weniger interessanten Philosophen aus verschiedenen Epochen. Die in den Texten formulierten Thesen werden im Präsenzunterricht erklärt und im Plenum diskutiert, wobei grosser Wert auf den Bezug zur Medizin gelegt wird. Jedes Modul geht von einer medizinischen Fragestellung aus. Zur Nachbereitung

\section{Selten habe ich einen Kurs erlebt, in dem die Teilnehmer über 8 Stunden pausenlos konzentriert bis zum Schluss bei der Sache waren.}

dem man plötzlich etwas in Frage stellen muss, das einem bisher klar und eindeutig erschien, und man verzweifelt nach überzeugenden Argumenten sucht, seine Glaubenssätze zu verteidigen. Ebenso wenig vor dem Moment, in dem man vom Philosophen sanft darauf hingewiesen wird, dass man den Text auch noch ganz anders verstehen kann. Wenn man die Hemmung, sich Verständnisschwierigkeiten einzugestehen, überwinden und sich den Mangel an geisteswissenschaftlicher Bildung verzeihen kann, ist dieser Kurs ein grosser Gewinn. Dass es den anderen Teilnehmenden genau gleich ergeht, schafft ein verbindendes Element.

\section{«Philosophie und Medizin» an der Univer- sität Luzern \\ Informationen zum CAS (Certificate of Advanced Studies) «Philosophie und Medizin» der Uni- versität Luzern erhalten Sie unter www.philo medizin.ch und bei der Studienleitung manuel. bachmann@unilu.ch. Der nächste Kurs beginnt im Juni 2011. Anmeldungen sind noch möglich.}

wird am Kurstag weiterführendes Material zur Vertiefung der Thematik verteilt. Wie es sich für einen Zertifikatskurs an einer Universität gehört, muss am Schluss eine rund 15-seitige Zertifikatsarbeit zu einem selbstgewählten Thema verfasst werden. Bei unserem bescheidenen philosophischen Vorwissen und der beschränkten Zeit sicher keine triviale Aufgabe, aber dank Coaching durch die Studienleitung machbar.

\section{Was bringt's?}

Die Skepsis breiter Bevölkerungskreise gegenüber der «Schulmedizin», die Dominanz finanzieller Gesichtspunkte über inhaltliche Aspekte in der Gesundheitsdiskussion sowie das Gefühl einer zunehmend schwierigeren Kommunikation zwischen den verschiedenen Partnern des Gesundheitswesens wird als Zeichen einer Krise der Medizin interpretiert [1]. Genau dieses Unbehagen angesichts einer solchen Krise konnte ich in den Diskussionen mit den Kursteilnehmern vielfach heraushören. Der Kurs «Philosophie und Medizin» trägt dazu bei, uns zu befähigen, den gesellschaftlichen Diskurs über Probleme und Aufgaben der Medizin aktiv mitzugestalten. 